\title{
Finding a Nash Equilibrium in Spatial Games is an NP-Complete Problem
}

Baron, Richard; Durieu, Jacques; Haller, Hans Hermann; Solal, Philippe

Publication date:

2002

Document version

Early version, also known as pre-print

Citation for published version (APA):

Baron, R., Durieu, J., Haller, H. H., \& Solal, P. (2002). Finding a Nash Equilibrium in Spatial Games is an NP. Complete Problem. Department of Economics, University of Copenhagen. 


\section{DISCUSSION PAPERS Department of Economics University of Copenhagen}

$$
\text { 02-19 }
$$

Finding a Nash Equilibrium in Spatial Games is an NP-Complete Problem

Richard Baron, Jaçques Durieu, Hans Haller, Philippe Solal

Studiestræde 6, DK-1455 Copenhagen K., Denmark

Tel. +45 35323082 - Fax +45 35323000

http://www.econ.ku.dk 


\title{
Finding a Nash Equilibrium in Spatial Games is an NP-Complete Problem*
}

RICHARD BARON ${ }^{a}$, JACQUES DURIEU $^{a}$, HANS HALLER $^{b, * *}$, PHILIPPE SOLAL $^{a}$

September 2002; revised, November 2002

${ }^{a}$ CREUSET, University of Saint-Etienne, 42100 Saint-Etienne, France.

${ }^{b}$ Department of Economics, Virginia Polytechnic Institute and State University, Blacksburg, VA 24061-0316, USA.

\begin{abstract}
We consider the class of (finite) spatial games. We show that the problem of determining whether there exists a Nash equilibrium in which each player has a payoff of at least $k$ is NP-complete as a function of the number of players. When each player has two strategies and the base game is an anti-coordination game, the problem is decidable in polynomial time.
\end{abstract}

Keywords: Spatial Games, NP-Completeness, Graph K-Colorability JEL Classification: C72.

*The hospitality of the Institute of Economics, University of Copenhagen, is gratefully acknowledged by the third author.

${ }^{*}$ Please address editorial correspondence to: Hans Haller, Department of Economics, Virginia Polytechnic Institute and State University, Blacksburg, VA 24061-0316, USA. E-mail: haller@vt.edu 


\section{Introduction}

Sahni (1974) showed that computing a pure Nash equilibrium in a finite $n$-player game in normal form is NP-complete as a function of the number of players. More recently, Gilboa and Zemel (1989) considered the related problem of computing a mixed Nash equilibrium. They showed that given a game $\mathcal{G}$ and an integer $k$, computing whether there exists a mixed Nash equilibrium where each player gets a payoff of at least $k$ is NP-complete as a function of the number of strategies while computing a correlated equilibrium where each player gets a payoff of at least $k$ is decidable in polynomial time. These results are the most basic complexity results concerning solution concepts for non-cooperative games in normal form ${ }^{1}$. They suggest that one should not expect to find a polynomial time algorithm for determining a Nash equilibrium in an arbitrary finite game in normal form. Thus if we expect to be able to compute a Nash equilibrium, we will have to restrict the instances to classes of games other than the class of arbitrary finite games in normal form. We have at our disposal a very large number of classes from which to choose.

Here we consider the class of spatial games. An $n$-player spatial game is a game in normal form where the payoff of each player is the weighted sum of the payoffs from playing each of his neighbors, where the set of neighbors of a player is given by the spatial structure of the game. Spatial games are studied by Blume (1993), Ellison (1993), Young (1998), and Baron et al. (2002), among others. This type of strategic interaction is of interest for economists because it arises quite naturally in social situations. Consider the following example. Players are firms where each firm is regarded as a repository of competencies. Represent each firm by the vertex of a graph and join two firms by an edge if they cooperate in a process of production. Thus the firms are organized in a network of two-firm cooperative agreements where a firm may cooperate with several firms. We say that two firms are neighbors if they cooperate in a process of production. Each firm has to choose an activity among a finite set of activities. Activities are complementary in the sense that they represent different phases of a process of production. Thus each firm prefers to choose an activity unlike the activities chosen by its partners. The total payoff of a player is the sum of the payoffs he gets from all his cooperative agreements. An obvious goal is to compute the maximum payoff the players can get at an equilibrium of this spatial game. We show

\footnotetext{
${ }^{1}$ For complexity considerations in extensive form games see Koller and Megiddo (1992), Koller, Megiddo, and von Stengel (1996), Chu and Halpern (2001). Conitzer and Sandholm (2002) strengthen and extend the results by Gilboa and Zemel. Furthermore, they obtain hardness results for games of incomplete information and stochastic games.
} 
that the problem of computing a Nash equilibrium, where each player gets a total payoff of at least $k$, is NP-complete as a function of the number of players. On the positive side, this problem becomes solvable in polynomial time when the number of strategies is two for each player. The presentation of the paper is as follows. In section 2 we define the spatial game and give a brief introduction to the theory of computational complexity. In section 3 we prove the NP-completeness result.

\section{Preliminaries}

\section{Spatial Games}

The basic building block is a two-player game $G$, called the base game, with a common finite action set $X$, and combined payoff function $\pi: X \times$ $X \rightarrow \mathbb{R}^{2}$ which assigns to each strategy profile $x=\left(x_{1}, x_{2}\right)$ the pair $\pi(x)=$ $\left(\pi_{1}(x), \pi_{2}(x)\right)$ of payoffs. We associate with $G$ a spatial game

$$
\mathcal{G}=\left(I, \Gamma_{n},\left(S_{i}\right)_{i \in I},\left(u_{i}\right)_{i \in I}\right)
$$

in the following way. The player set is $I=\{1, \ldots, n\}$. Every player $i \in I$ has strategy set $S_{i}=X$. Moreover, the elements of $I$ form the vertices of a weighted graph $\Gamma_{n}$ of order $n$, with the interpretation that player $i$ is located at vertex $i$. $E$ denotes the set of edges of $\Gamma_{n}$. Two vertices or players $i$ and $j$ are neighbors, if $\{i, j\} \in E . N(i)$ denotes the set of neighbors of $i . \Gamma_{n}$ is undirected in the sense that $j \in N(i)$ if and only if $i \in N(j)$. Furthermore, we assume that $N(i) \neq \emptyset$ for all $i$, i.e. none of the vertices is isolated. Each $\{i, j\} \in E$ has a weight $w_{i j}$ that measures its relative importance. Note that $w_{i j}$ is not necessarily equal to $w_{j i}$. Payoffs in the spatial game $\mathcal{G}$ are given by

$$
u_{i}(s)=\sum_{j \in N(i)} w_{i j} \pi_{i}\left(s_{i}, s_{j}\right)
$$

for $i \in I, s=\left(s_{j}\right)_{j \in I} \in \prod_{j \in I} S_{j} \equiv \mathcal{S}$, that is, a player collects the aggregate weighted payoffs from playing with each of his neighbors.

Let $s^{*} \in \mathcal{S}$ be a stragegy profile of the spatial game $\mathcal{G}$. The profile $s^{*}$ is a Nash equilibrium if for all $i \in I, s_{i} \in S_{i}$,

$$
u_{i}\left(s^{*}\right) \geq u_{i}\left(s_{i}, s_{-i}^{*}\right)
$$

where $s_{-i}^{*}=\left(s_{j}^{*}\right)_{j \neq i}$. Clearly, $w_{i j}=w_{j i}=0$ has the same effect on payoffs as $\{i, j\} \notin E$. We can set $w_{i j}=w_{j i}=0$ for $\{i, j\} \notin E$ and assume without loss of generality that $w_{i j}>0$ or $w_{j i}>0$ for $\{i, j\} \in E$. Then $\{i, j\} \in E$ if and only if $w_{i j}+w_{j i}>0$. That is $E$ is determined by the weight matrix 
$W=\left[w_{i j}\right]$. Hence for given $X$ and $n$, the variable inputs for an instance of the spatial game consist of the entries of the weight matrix $W=\left[w_{i j}\right]$.

NP-Completeness

For a comprehensive introduction to NP-completeness the reader is referred to Garey and Johnson (1979). Let $\mathbf{P}$ denote the class of problems that can be solved on a deterministic Turing machine by a polynomial time algorithm, that is, polynomial in the length of inputs for an instance of the problem. Let NP denote the class of all decision problems which can be solved in polynomial time by a nondeterministic Turing machine. Instead of using the notion of nondeterminism, one can define the class NP in terms of the concept of polynomial-time verification. A verification algorithm is an algorithm which takes as input an instance of the problem and a candidate solution to the problem, called a certificate, and verifies in polynomial time whether the certificate is a solution to the given instance. Thus the class NP is the class of problems which can be verified in polynomial time.

The fundamental open question in computational complexity is whether $\mathbf{P}=\mathbf{N P}$. By definition $\mathbf{P} \subset \mathbf{N P}$. It is not known, however, whether all problems in NP can, in fact, be solved in polynomial time by a deterministic Turing machine. The generally accepted belief is that $\mathbf{P} \neq \mathbf{N P}$. In an effort to determine whether $\mathbf{P}=\mathbf{N P}$, the class of NP-complete problems has been introduced. We say that a problem $P_{1}$ is polynomial-time reducible to a problem $P_{2}$, written $P_{1} \preceq_{p} P_{2}$, if

(i) there exists a function $f$ which maps any instance of $P_{1}$ to an instance of $P_{2}$ in such a way that $I_{1}$ is a "yes" instance of $P_{1}$ if and only $f\left(I_{1}\right)$ is a "yes" instance of $P_{2}$.

(ii) for any instance $I_{1}$, the instance $f\left(I_{1}\right)$ can be constructed in polynomial time.

If $P_{1}$ is polynomial-time reducible to $P_{2}$, we can say that any algorithm for solving $P_{2}$ can be used to solve $P_{1}$. Intuitively, problem $P_{1}$ is "no harder" to solve than problem $P_{2}$. A problem $P$ is said to be NP-complete if $(i)$ $P \in \mathbf{N P}$, and (ii) for every problem $P^{\prime} \in \mathbf{N P}, P^{\prime} \preceq_{p} P$. If a problem satisfies condition $(i i)$ but not necessarily condition $(i)$, then we say that it is NP-hard. Let NPc denote the class of NP-complete problems.

The binary relation $\preceq_{p}$ is transitive on the set of decision problems. Because of this, a method frequently used in demonstrating that a given problem is 
NP-complete is the following:

(i) show that $P \in \mathbf{N P}$, and

(ii) show there exists a problem $P^{\prime} \in \mathbf{N P c}$, such that $P^{\prime} \preceq_{p} P$.

It follows from the definition of NP-completeness that if any problem in NPc can be solved in polynomial time, then every problem in NPc can be solved in polynomial time, and $\mathbf{P}=\mathbf{N P}$. On the other hand, if there is some problem in NPc that cannot be solved in polynomial time, then no problem in NPc can be solved in polynomial time.

\section{The NP-Completeness Result}

We show that the problem of determining a Nash equilibrium in a spatial game is NP-complete as a function of the number of players. To state this problem in the accepted format, we convert it to a decision problem in considering the problem of deciding whether the Nash profile gives a payoff of at least $k \in \mathbb{N}$.

NASH FOR SPATIAL GAMES (NSG)

INSTANCE: A finite spatial game $\mathcal{G}=\left(I, \Gamma_{n},\left(S_{i}\right)_{i \in I},\left(u_{i}\right)_{i \in I}\right)$ and a positive integer $k$. The finite spatial game is given by $X, n$, and a weight matrix $W=\left[w_{i j}\right]$.

QUESTION: Does there exist a Nash equilibrium in $\mathcal{G}$ in which each player obtains the payoff of at least $k$ ?

Proposition $1 N S G \in$ NPc.

Proof: We follow the method described in section 2. We must do two things. First we must show that NSG $\in$ NP. The nondeterministic Turing machine just guesses an arbitrary strategy profile $s \in \mathcal{S}$ and has to consider $n \cdot(|X|-1)$ deviations and to take the $n(n-1)$ weights into account in the computation in order to verify whether the Nash equilibrium and payoff conditions are satisfied at $s$ where the time of computing a single payoff is of the order $n$. Second we must construct a reduction from a known NP-complete problem to NSG. We use the GRAPH $k$-COLORABILITY problem.

\section{GRAPH $k$-COLORABILITY}

INSTANCE: A graph $\Gamma_{n}=(V, E)$ and a positive integer $k \leq|V|$.

QUESTION: Is $\Gamma_{n} k$-colorable, i.e. does there exist an assignment of $k$ 
colors $\{1,2, \ldots, k\}$ to the vertices of $\Gamma_{n}$ so that neighboring vertices are assigned different colors ? This problem is NP-complete for an arbitrary $k$.

Given an instance $\Gamma_{n}=(V, E)$ and $k$ of GRAPH $k$-COLORABILITY, we construct an instance $\mathcal{G}$ of NSG as follows. In a FIRST STEP, we restrict ourselves to graphs $\Gamma_{n}=(V, E)$ without isolated points. The set of vertices constitutes the set of players. The neighborhood of player $i$ is exactly the set of vertices adjacent to vertex $i$, i.e. $N(i)=\{j \in V:\{i, j\} \in E\}$ and $N(i) \neq \emptyset$ if $i$ is not isolated. For distinct players $i$ and $j$ in $V$, define the weights $w_{i j}$ as follows:

$$
w_{i j}=w_{j i}= \begin{cases}1 & \text { if }\{i, j\} \in E \\ 0 & \text { otherwise }\end{cases}
$$

The finite set of strategies is $S_{i}=X=\{1, \ldots, k\}$ for every player $i \in V$. The base game payoff of player $i$ when he encounters a player $j \in N(i)$ is given by

$$
\pi_{i}\left(s_{i}, s_{j}\right)= \begin{cases}k /|N(i)| & \text { if } s_{i} \neq s_{j} \\ 0 & \text { otherwise }\end{cases}
$$

We must show that an instance of GRAPH $k$-COLORABILITY is a "yes" instance if and only if the constructed game $\mathcal{G}$ has a Nash equilibrium in which each player gets a payoff of at least $k$.

First assume that $\Gamma_{n}$ is $k$-colorable. Then no two neighboring vertices are assigned the same color. Create a strategy profile $s^{*}$ as follows : $s_{i}^{*}=c_{i}$ where $c_{i}$ is the color assigned to vertex $i$. The strategy profile $s^{*}$ is a Nash equilibrium in which each player gets a payoff of at least $k$ for $\mathcal{G}$ because $(i)$ each player $i$ gets the maximum payoff $k /|N(i)|$ in each bilateral encounter, (ii) his total payoff is $k$.

Conversely, suppose that $\mathcal{G}$ has a Nash equilibrium $s^{*}$ in which each player gets a payoff of at least $k$. Notice that the maximum total payoff the players can get in this game is in fact $k$. Each player gets a total payoff of $k$ only if he gets $k /|N(i)|$ in each bilateral encounter i.e. only if for each pair of neighbors, the players choose a different strategy. We can therefore create a "yes" instance of GRAPH $k$-COLORABILITY.

It should be clear that our construction for creating an instance of NSG from an instance of GRAPH $k$-COLORABILITY, can be carried out in polynomial time. The length of an instance of GRAPH $k$-COLORABILITY is $\mathcal{O}(n+|E|+k)$. The graph of the constructed spatial game is exactly $\Gamma_{n}$, the cardinality of the common strategy set is $k$, and the base game payoff can be determined with the elements of $\Gamma_{n}$. In particular, $k /|N(i)|$ can be computed when the graph structure is given. Therefore, an instance of NSG 
can be constructed from an instance of GRAPH $k$-COLORABILITY in polynomial time. Let $f_{2}$ denote the above mapping that associates instances of GRAPH $k$-COLORABILITY for graphs without isolated points and NSG.

In a SECOND STEP we associate to any instance of GRAPH $k$-COLORABILITY an instance of GRAPH $k$-COLORABILITY without isolated points. Denote this mapping $f_{1} . f_{1}$ is constructed as follows. $k$ remains unchanged. If $\Gamma_{n}=(V, E)$ is any graph, its image assumes the form $\Gamma_{n}^{*}=\left(V, E^{*}\right)$. In case $\Gamma_{n}$ has no isolated points, set $E^{*}=E$. In this case, $\Gamma_{n}=\Gamma_{n}^{*}$ and $\Gamma_{n}$ is $k$-colorable if and only if $\Gamma_{n}^{*}$ is. In case $\Gamma_{n}$ does have isolated points, let $J$ denote the set of isolated points and label them $j_{1}, \ldots, j_{m}$. In the special case $J=V$, set $E^{*}=\left\{\left\{j_{1}, j_{2}\right\},\left\{j_{2}, j_{3}\right\}, \ldots,\left\{j_{m-1}, j_{m}\right\}\right\}$. Then both $\Gamma_{n}$ and $\Gamma_{n}^{*}$ are $k$-colorable in the following way: Choose color $c_{r} \equiv r \bmod k$ for node $j_{r}, r=1, \ldots, m$. In the subcase $J \neq V$, choose any $j_{0} \in V \backslash J$ and set $E^{*}=E \cup\left\{\left\{j_{0}, j_{1}\right\},\left\{j_{1}, j_{2}\right\}, \ldots,\left\{j_{m-1}, j_{m}\right\}\right\}$. Obviously, if $\Gamma_{n}^{*}$ is $k$-colorable, then $\Gamma_{n}$ is also $k$-colorable, since $E \subset E^{*}$. Suppose $\Gamma_{n}$ is $k$-colorable. Then $\Gamma_{n}^{*}$ is $k$-colorable as well. Namely, fix any $k$-coloring of $\Gamma_{n}$ and denote by $c_{0}$ the color given to node $j_{0}$ in that coloring. Keep the colors of nodes in $V \backslash J$. Assign color $c_{r} \equiv c_{0}+r \bmod k$ to node $j_{r}, r=1, \ldots, m$. Then the new coloring is a $k$-coloring of $\Gamma_{n}^{*}$. Hence we have constructed a mapping $f_{1}$ that assigns to any graph $\Gamma_{n}$ a graph $\Gamma_{n}^{*}$ without isolated points such that $\Gamma_{n}$ is $k$-colorable if and only if $\Gamma_{n}^{*}$ is. The identification and labeling of isolated points plus the construction of the chain $\left\{\left\{j_{0}, j_{1}\right\},\left\{j_{1}, j_{2}\right\}, \ldots,\left\{j_{m-1}, j_{m}\right\}\right\}$ can be achieved in polynomial time. The composition $f=f_{2} \circ f_{1}$ constructs in polynomial time a corresponding NSG instance from any GRAPH $k$-COLORABILITY instance.

\section{End of Proof.}

\section{Comments:}

1. The GRAPH $k$-COLORABILITY problem is known to be in $\mathbf{P}$ when $k=2$. Therefore, the NSG problem is in $\mathbf{P}$, when each player has two strategies and the base game is an anti-coordination game (that is a base game in which players prefer to choose a strategy unlike the strategy chosen by their opponents).

2. In the main step of the proof, the constructed NSG instance satisfies symmetry of the weights, $w_{i j}=w_{j i}$. Therefore, NSG remains NPc when restricted to the subclass of spatial games with symmetric weights.

3. The construction in the last step of the proof can be modified to show that GRAPH $k$-COLORABILITY remains NPc when restricted to connected graphs. 
4. Proposition 1 forms a contrast to the results obtained by Gilboa and Zemel (1989) and Mailath, Samuelson and Shaked (1997). As underlined in the introduction, Gilboa and Zemel (1989) showed that computing a correlated equilibrium where each player gets a payoff of at least $k$ is decidable in polynomial time. On the other hand Mailath, Samuelson and Shaked (1997) showed that a correlated equilibrium is equivalent to a Nash equilibrium of a specific spatial game. This equivalence can be implemented in polynomial time by simple manipulation of a set of equations. Proposition 1 still remains

valid, since the Mailath-Samuelson-Shaked model of local interaction is quite different from ours.

\section{References}

Baron R., Durieu J., Haller H., Solal P. (2002) "Control Costs and Potential Functions for Spatial Games", Working Paper, Virginia Polytechnic Institute and State University, Blacksburg, VA.

Chu F., Halpern J. (2001), "On the NP-Completeness of Finding an Optimal Strategy in Games with Common Payoffs", International Journal of Game Theory, 30:99-106.

Blume L.E. (1993) "Statistical Mechanics of Strategic Interaction", Games and Economic Behavior, 5:387-426.

Conitzer, V., Sandholm, T. (2002), "Complexity Results about Nash Equilibria", Computer Science Department, Carnegie Mellon University, Pittsburgh, PA.

Ellison, G. (1993), "Learning, Local Interaction, and Coordination", Econometrica, 61:1047-1071.

Garey M.R., Johnson D.S. (1979), Computers and Intractability. A Guide to The Theory of NP-Completeness, Freeman, San Fransico.

Gilboa I., Zemel E. (1989), "Nash and Correlated Equilibria: Some Complexity Considerations", Games and Economic Behavior, 1:80-93.

Koller D., Megiddo N. (1992), "The Complexity of Two-Person Zero-Sum Games in Extensive Form", Games and Economic Behavior, 4:528-552.

Koller D., Megiddo N., von Stengel B. (1996), "Efficient Computation of Equilibria for Extensive Two-Person Games", Games and Economic Behavior 14:247-259. 
Mailath G.J., Samuelson L., Shaked A. (1997), "Correlated Equilibria and Local Interactions", Economic Theory, 9:551-556.

Sahni S. (1974), "Computationally Related Problems", SIAM Journal of Computing, 3:262-279.

Young H.P. (1998) Individual Strategy and Social Structure, Princeton University Press, Princeton, New Jersey. 